\title{
Dominance shifts increase the likelihood of soft selective sweeps
}

\author{
Pavitra Muralidhar ${ }^{1,2, *} \quad$ Carl Veller ${ }^{2}$
}

\begin{abstract}
Genetic models of adaptation to a new environment have typically assumed that the alleles involved maintain a constant fitness dominance across the old and new environments. However, theories of dominance suggest that this should often not be the case. Instead, the alleles involved should frequently shift from recessive deleterious in the old environment to dominant beneficial in the new environment. Here, we study the consequences of these expected dominance shifts for the genetics of adaptation to a new environment. We find that dominance shifts increase the likelihood that adaptation occurs from the standing variation, and that multiple alleles from the standing variation are involved (a soft selective sweep). Furthermore, we find that expected dominance shifts increase the haplotypic diversity of selective sweeps, rendering soft sweeps more detectable in small genomic samples. In cases where an environmental change threatens the viability of the population, we show that expected dominance shifts of newly beneficial alleles increase the likelihood of evolutionary rescue and the number of alleles involved. Finally, we apply our results to a well-studied case of adaptation to a new environment: the evolution of pesticide resistance at the Ace locus in Drosophila melanogaster. We show that, under reasonable demographic assumptions, the expected dominance shift of resistant alleles causes soft sweeps to be the most frequent outcome in this case, with the primary source of these soft sweeps being the standing variation at the onset of pesticide use, rather than recurrent mutation thereafter.
\end{abstract}

\footnotetext{
${ }^{1}$ Center for Population Biology, University of California, Davis, CA 95616

${ }^{2}$ Department of Evolution and Ecology, University of California, Davis, CA 95616

*pmuralidhar@ucdavis.edu
} 


\section{Introduction}

The primary concern of evolutionary genetics is to understand the genetic processes that underlie organisms' adaptation to their environments. An important goal in this field is therefore to understand the nature of the genetic variation from which adaptation to a new environment typically occurs. When adaptation to a new environment is partly or wholly due to fixation of a newly beneficial allele at a given locus (a 'selective sweep'), the question arises whether this fixation typically proceeds from a single initial copy of the beneficial allele (a 'hard selective sweep') or from multiple distinct copies that were possibly already segregating at the time of the environmental change (a 'soft selective sweep') (Hermisson and Pennings 2005; Pritchard et al. 2010; Messer and Petrov 2013). The relative frequency of hard versus soft sweeps has been the subject of much recent discussion [e.g., Messer and Petrov (2013); Jensen (2014); Schrider and Kern (2017); Hermisson and Pennings (2017); Harris et al. (2018)].

In the classic model of a selective sweep in response to a change of environment, a mutation that was neutral or deleterious before the environmental change becomes beneficial after the environmental change (Orr and Betancourt 2001). This simple model has been studied intensively from the perspective of mathematical population genetics [e.g., Hermisson and Pennings (2005); Pennings and Hermisson (2006); Pritchard et al. (2010); Messer and Petrov (2013); Hermisson and Pennings (2017); Stephan (2019)], and has served as the theoretical foundation for much empirical work [e.g., Barrett and Schluter (2008); Messer and Petrov (2013); Garud et al. (2015); Schrider and Kern (2017)].

An assumption that is usually invoked in both theoretical and empirical studies of this model is that the fitness dominance of the focal allele is invariant across the environmental change - that is, the allele's dominance with respect to its deleterious effect in the old environment is equal to its dominance with respect to its beneficial effect in the new environment [an exception is Orr and Betancourt (2001), discussed below]. The reason for this assumption is convenience: it simplifies theoretical calculations and buys a degree of freedom in empirical studies. However, it also sidesteps a rich, century-old literature on physiological and evolutionary theories of allelic dominance.

Physiological theories of dominance provide mechanistic explanations for the observation that loss-of-function mutations are typically recessive with respect to the wild-type allele, while gain-offunction mutations are typically dominant. De Vries and Bateson pioneered this literature in the late 19th century (Falk 2001), but its most famous representation is in the Wright-Kacser-Burns theory of metabolic dominance (Wright 1934; Kacser and Burns 1981; Keightley 1996), which, by explicitly modeling the chemistry of metabolic pathways, showed that their operation is intrinsically robust to single loss-of-function (or decrease-of-function) mutations. Physiological theories of dominance have generally focused on genes encoding enzymes or other products with 'quasi-catalytic' properties. However, empirical work has shown that the predictions of the physiological theories in fact hold across a much broader set of gene categories, suggestive of a need for more general explanations of patterns of dominance (Phadnis and Fry 2005; Agrawal and Whitlock 2011).

Such explanations can be found in evolutionary theories of dominance, which seek to explain why beneficial alleles tend to be dominant while deleterious alleles tend to be recessive. This literature begins in the 1920s with Fisher's mathematical demonstration that modifiers of an allele's dominance are under positive selection to increase its dominance when it is beneficial and to decrease its dominance when it is deleterious (Fisher 1928). While Fisher's treatment was abstract, subsequent work - in many cases guided by physiological theories of dominance - has developed more mechanistically-explicit evolutionary theories of dominance, based on, for example, the dynamics of metabolic pathways [reviewed in Bourguet (1999)], models of optimal gene expression (Hurst and Randerson 2000), and multidimensional fitness landscapes (Manna et al. 2011).

In a selective sweep after a change of environment, there are two distinct scenarios under which the focal allele transitions from deleterious before the environmental change to beneficial after. In the first scenario, it is the same phenotype that is primarily under selection before and after the environmental 
change, but the direction of selection on this phenotype changes. For example, an allele that reduces limb length would be deleterious in an environment in which long limbs are favored, but would become beneficial in a new environment where short limbs are advantageous [e.g., Donihue et al. (2018)]. In this scenario, the focal allele's fitness dominance is governed by its dominance with respect to the selected phenotype; since the selected phenotype does not change across the environmental shift, nor would we expect the focal allele's fitness dominance to change significantly.

In the second scenario, the environmental change corresponds to a change in the phenotype that is primarily under selection. In this case, the focal allele's effect on heterozygotes' fitness is modulated through different phenotypes before and after the environmental change, and so the allele's fitness dominance is not expected to remain constant. In fact, the evolutionary and physiological theories of dominance predict that the dominance of the focal allele should shift from recessive when it is deleterious to dominant when it is beneficial.

For a concrete example of this second scenario, consider the Ace locus in insects, where recent adaptation has occurred in response to pesticide use, forming an important case study in the selective sweeps literature [e.g., Karasov et al. (2010); Garud et al. (2015)] and evolutionary genetics more broadly [e.g., Bourguet et al. (1997); Lenormand et al. (1999)]. Ace encodes the enzyme acetylcholinesterase, which catalyzes acetylcholine at the neuromuscular junction (Hoffmann et al. 1992; Fournier and Mutero 1994; Mutero et al. 1994; Bourguet and Raymond 1998). Organophosphate pesticides, introduced in the mid-twentieth century, inhibit acetylcholinesterase by targeting its binding site (Fournier et al. 1993; Fournier and Mutero 1994; Mutero et al. 1994; Shi et al. 2004). Mutations at the Ace locus that alter the shape of the binding site can confer resistance to pesticide binding (Menozzi et al. 2004; Shi et al. 2004), and therefore can be beneficial in environments where pesticides are used (Bourguet and Raymond 1998). However, the reconfigured enzymes are intrinsically less efficient at binding acetylcholine itself (Hoffmann et al. 1992; Fournier and Mutero 1994), rendering them deleterious in pesticide-free environments [as has been demonstrated in Drosophila melanogaster (Shi et al. 2004)]. Thus, in some geographic regions, these 'resistant' mutations were deleterious before the onset of pesticide use and beneficial after, conforming to the classic model described above. Moreover, the phenotype that was primarily under selection also changed, from intrinsic enzymatic efficiency before the onset of pesticide use, to the ability to avoid pesticide binding after. Consistent with the prediction of theories of dominance, the beneficial effect of these alleles in pesticide environmentsstemming from their ability to evade pesticide binding - has been shown generally to be dominant across insects (Bourguet and Raymond 1998; Charlesworth 1998). While the dominance of the deleterious effect of these alleles in pesticide-free environments - stemming from their reduced enzymatic efficiency - has not been directly measured in D. melanogaster, measurement of the activity of the enzyme produced by the resistant allele (Bourguet and Raymond 1998; Shi et al. 2004), together with evidence from other insect species (Zhang et al. 2015), suggests that it is likely to be recessive.

If, as we expect, it is often the case that adaptation to a new environment involves a change in the phenotype primarily under selection, then the alleles involved in this adaptation likely often transition from recessive deleterious in the old environment to dominant beneficial in the new environment. This fact obviously holds major implications for the genetics of adaptation to new environments. Intuitively, if the alleles underlying adaptation to a new environment were recessive deleterious beforehand, they will tend to have been present in greater numbers in the standing variation at the time of the environmental change, increasing the chance that multiple alleles were involved in the subsequent selective sweep. That is, the pattern of dominance shifts predicted by the physiological and evolutionary theories of dominance is expected to increase the relative likelihood of soft versus hard selective sweeps, as well as the importance in sweeps of alleles that were present in the standing variation at the time of the environmental change (versus those produced by mutation after the environmental change). Here, we carry out a quantitative investigation of the effect of these dominance shifts on the genetics of selective sweeps. 


\section{Methods}

The model. We study the classic model of a selective sweep in response to a change in the selective environment, adopting the framework set out by Hermisson and Pennings (2005). At a given locus, there are two alleles: the wild-type $A$ and the mutant $a$. At a discrete point in time, $T$, there is a sudden environmental change. Prior to $T$, the mutant allele $a$ was deleterious, with the relative fitnesses of genotypes $A A, A a$, and aa being $1,1-h_{d} s_{d}$, and $1-s_{d}$, such that $h_{d}$ is the fitness dominance of $a$ prior to $T$. After $T$, $a$ becomes beneficial, with the relative fitnesses of genotypes $A A$, $A a$, and $a a$ being $1,1+h_{b} s_{b}$, and $1+s_{b}$, such that $h_{b}$ is the fitness dominance of $a$ after $T$.

The population is of constant size $N$ (=10,000 in all simulations), and evolves according to a Wright-Fisher process. Prior to $T$, the alleles $A$ and $a$ mutate to one another at a constant, symmetric rate $u$ per replication. After $T$, there is no mutation, allowing us more precisely to study the likelihood and nature of adaptation from the standing variation (although we do later consider recurrent mutation after $T$ ).

Definition of a soft sweep. Several definitions of a 'soft sweep' exist in the literature (Hermisson and Pennings 2017). These definitions can be partitioned according to two axes. First, some definitions consider the ancestry of the entire population of alleles after the sweep, while others consider the ancestry of only a sub-sample. Second, for a sweep from the standing variation to be called soft, some definitions require only that multiple alleles present at the time of the environmental shift have descendants upon completion of the sweep, while other definitions further require that those ancestral alleles have distinct mutational origins [this axis distinguishes 'single-origin' and 'multiple-origin' soft sweeps in the terminology of Hermisson and Pennings (2017)].

We primarily employ a definition that uses the first option along each of these two axes. By this definition, a sweep from the standing variation is soft if multiple copies of the allele that were present at the time of the environmental shift have descendants among the entire population of alleles upon completion of the sweep. We call this a 'population' definition of soft sweeps. However, some of our results relate directly to the empirical detectability of soft sweeps - see, e.g., 'Measuring the haplotypic diversity of a sweep' below. For these results, we employ a 'sample' definition of soft sweeps, using the second option along the two axes above. By this definition, a sweep is soft if, in a given subsample of alleles at the time of fixation, there are multiple mutational origins (and therefore multiple distinguishable haplotypes).

Note that the sample-based definition of a soft sweep is stricter than the population-based definitionfewer sweeps will be classified as soft under the sample definition. Thus, the population and sample definitions provide liberal and conservative predictions of the number of soft sweeps expected to occur, which could therefore be interpreted as upper and lower bounds.

Simulation setup. We first characterize the mutation-selection-drift frequency distribution of $a$ before $T$ under various configurations of the parameters $u, s_{d}$, and $h_{d}$. For each configuration, we start in Hardy-Weinberg equilibrium at the focal locus, with the frequency of $a$ equal to its expectation $\left(u /\left[h_{d} s_{d}\right]\right.$ when $h_{d}>0$, and $u / \sqrt{s_{d}}$ when $\left.h_{d}=0\right)$. From this starting point, we allow the population to evolve for an initial burn-in period of $10^{6}$ generations, and thereafter record the frequency of each genotype in every generation for $10^{7}$ generations. The distribution across generations of these genotype frequencies constitutes our empirical mutation-selection-drift distribution. In addition to recording the genotype frequencies in each generation, we record each distinct mutational origin of an $a$ allele and the number of its descendant copies among $A a$ and $a a$ genotypes.

We then study adaptation after $T$. For a given configuration of the parameters $\left(u, s_{d}, h_{d}, s_{b}, h_{b}\right)$, we carry out 10,000 replicates of the following simulation: First, we randomly draw a set of starting genotype frequencies from the before- $T$ mutation-selection-drift distribution for $\left(u, s_{d}, h_{d}\right)$, estimated 
as described above. If one or more copies of $a$ are present in this initial genotype configuration, we tag each separate copy, and track the descendants of each of these copies in every subsequent generation. The simulation ends when $a$ fixes or goes extinct.

For each parameter configuration, we calculate the proportion of trials that result in each of the four possible outcomes: (i) No standing variation. No copies of $a$ were present in the initial genotype configuration. (ii) Failed sweep. There was at least one copy of $a$ present in the initial genotype configuration, but $a$ subsequently went extinct. (iii) Hard sweep. $a$ fixes, and all copies of $a$ at the time of fixation descend from a single ancestral copy in the initial genotype configuration. (iv) Soft sweep. $a$ fixes, and the copies of $a$ at the time of fixation descend from more than one copy in the initial genotype configuration. Note that this analysis employs the 'population definition' of a soft sweep.

In addition, in those trials in which a sweep occurred, we calculate the number of distinct mutational origins present among the swept alleles, and the frequencies of these distinct mutations.

Measuring the haplotypic diversity of a sweep. When a sweep has occurred from alleles that were present in the standing variation, we are interested in the haplotypic diversity of the sweep. The haplotypic diversity of a sweep is interesting not just from a theoretical perspective, but also for a practical empirical reason: when a soft sweep is mutationally more diverse, we have a better chance of being able to recognize, in a finite sample of sequenced alleles, that a soft sweep has occurred. Therefore, in measuring the haplotypic diversity of sweeps, we shall aim to use metrics with practical relevance to the empirical assessment of selective sweeps. In relating these metrics to the empirical assessment of soft sweeps, we shall use the 'sample definition' of a soft sweep, that the alleles involved have multiple mutational origins.

Suppose that, in a trial in which $a$ sweeps to fixation, the alleles present upon completion of the sweep derive from $m$ distinct mutations before $T$. We record $m$. Let the frequency of the descendants of ancestral mutation $i$ at the time of fixation be $p_{i}, i=1, \ldots, m$. First, for various $n$, we measure the mutational (or 'haplotypic') diversity of the sweep by calculating the Gini-Simpson diversity index of order $n:{ }^{n} \mathrm{x}=1-\sum_{i=1}^{m} p_{i}^{n}$ [modified from Jost (2006)]. Empirically, ${ }^{n} \mathrm{x}$ is the probability that, in a random sample of $n$ alleles taken from the population at the time of fixation, there are at least two distinct mutational origins and therefore at least two distinct haplotypes - i.e., ${ }^{n} \mathrm{x}$ is the probability that a soft sweep from the standing variation can be detected in a random sample of $n$ descendant alleles. Second, we record, for each value of $n$, the expected number of ancestral mutations represented in a random sample of $n$ descendant alleles at the time of fixation, $\mathbb{E}[\#$ ancestors $\mid n]=m-\sum_{i=1}^{m}\left(1-p_{i}\right)^{n}$. The formulae above are valid under the assumption that $n \ll N$.

Recurrent mutation. Selective sweeps can derive from copies of $a$ present in the standing variation at the time of the environmental change, or from copies of $a$ that appeared by mutation after the environmental change. To incorporate the possibility of recurrent mutation after the environmental change, we keep the symmetric $A \leftrightarrow a$ mutation rate equal before and after the environmental change. Because $a \rightarrow A$ mutation after the environmental change generates a perpetual supply of $A$ alleles, we say that $a$ has 'fixed' (and a selective sweep has occurred) when it achieves a frequency equal to 0.99 times the mean of its mutation-selection-drift distribution when beneficial.

Recurrent $A \rightarrow a$ mutation guarantees that a selective sweep will eventually occur after the environmental change. Therefore, we restrict our analysis of recurrent mutation to cases where a selective sweep occurs that involves alleles from the standing variation. We measure, in these cases, what proportion of $a$ alleles present at the time of fixation derive from mutations that appeared after the environmental change versus mutations that were present in the standing variation at the time of the environmental change. Owing to the greater computational expense of these simulations, only 5,000 trials were run for some of the parameter configurations. 
Evolutionary rescue. The environmental change at time $T$ could be such that the population would go extinct in the absence of the newly beneficial allele $a$. The question of a selective sweep of $a$ is then one of evolutionary rescue. To study this situation requires abandoning our previous assumption of a constant population size, and explicitly modeling how the population shrinks or grows as a function of its genotypic composition.

We assume that the population is characterized by an intrinsic reproductive rate, $r_{0}$. An interpretation of $r_{0}$ is that, in a sexual population, and in the absence of any selective or ecological constraints, each individual would have $2\left(1+r_{0}\right)$ successful offspring on average; the population would then grow at rate $r_{0}$ per generation. We scale 'absolute' fitnesses according to $r_{0}$, such that an absolute fitness of $w$ implies an expectation of $2\left(1+r_{0}\right) w$ successful offspring in the absence of any ecological constraints. The full population dynamics is then determined as follows. Suppose that, in generation $t$, the population is of size $N_{t}$ and the average absolute fitness of its members is $\bar{w}$. The 'unconstrained' population size in the next generation, $N_{t+1}^{*}$, is a Poisson random variable with mean $\left(1+r_{0}\right) \bar{w} N_{t}$; the actual population size in the next generation, $N_{t+1}$, is the smaller of $N_{t+1}^{*}$ and the carrying capacity $K=10,000$. Once $N_{t+1}$ is decided, the genotypic composition of generation $t+1$ is determined by randomly drawing parental alleles from generation $t$, independently for each allele in generation $t+1$, and with probabilities proportional to the fitnesses of the individuals carrying the alleles in generation $t$. Notice that, in the case where $N_{t+1}=N_{t+1}^{*}$, the 'top down' model described above corresponds to a simple 'bottom up' model where mating is random and the number of alleles contributed to generation $t+1$ by an individual in generation $t$ with absolute fitness $w$ is a Poisson random variable with mean $2\left(1+r_{0}\right) w$.

The general scenario for evolutionary rescue that we wish to model has the following key features: (i) Before $T$, the absolute fitness of the $A A$ genotype, $w_{A A}$, is such that $\left(1+r_{0}\right) w_{A A}>1$, so that a population fixed (or nearly so) for $A$ is held at its carrying capacity. (ii) After $T$, the absolute fitness of the $A A$ genotype, $w_{A A}^{\prime}$, is such that $\left(1+r_{0}\right) w_{A A}^{\prime}<1$, so that a population fixed for $A$ would decline exponentially to extinction. (iii) After $T$, the absolute fitness of the $a a$ genotype, $w_{a a}^{\prime}$, is such that $\left(1+r_{0}\right) w_{a a}^{\prime}>1$, so that a population fixed for $a$ would increase exponentially to the carrying capacity $K$. (iv) $a$ is deleterious before $T$ because of some impairment of basic function relative to $A$. After $T, a$ confers resistance to whatever new selective force is threatening the population's survival, but it still carries the cost of its impaired basic function. Therefore, $w_{a a}^{\prime}<w_{A A}$.

To incorporate the above scenario into our model of selective sweeps, we assume that, before $T$, the absolute fitnesses of the genotypes $A A, A a$, and $a a$ are $1,1-h_{1} s_{1}$, and $1-s_{1}$ respectively, where $s_{1}$ is the absolute fitness cost to $a a$ individuals because of the impaired function of $a$, and $h_{1}$ is the dominance of $a$ relative to $A$ with respect to this impaired function. Criterion (i) then simply requires that $r_{0}>0$. After $T$, the absolute fitnesses of the genotypes $A A, A a$, and $a a$ are $1-s_{2}$, $\left(1-h_{2} s_{2}\right)\left(1-h_{1} s_{1}\right)$, and $1-s_{1}$ respectively, where $s_{2}$ is the absolute fitness cost to $A A$ individuals because of $A$ 's deleterious effect in the new environment (which $a$ does not suffer), and $h_{2}$ is the dominance of $A$ relative to $a$ with respect to this new deleterious effect. Criteria (ii) and (iii) require that $\left(1+r_{0}\right)\left(1-s_{2}\right)<1$ and $\left(1+r_{0}\right)\left(1-s_{1}\right)>1$, which in turn requires that $s_{2}>s_{1}$, i.e., that the deleterious effect of $A$ in the new environment is more severe than the deleterious effect of $a$ in the old environment.

For each configuration of the parameters $\left(u, s_{1}, h_{1}, s_{2}, h_{2}, r_{0}\right)$, we carry out 10,000 replicates of the following simulation: First, we randomly draw a starting set of genotype frequencies from the before$T$ empirical mutation-selection-drift distribution corresponding for $\left(u, s_{1}, h_{1}\right)$, estimated as described above. If one or more copies of $a$ are present in this initial genotype configuration, we tag each separate copy, and track the descendants of each of these copies in every subsequent generation. We allow the population size and genotype frequencies to evolve according to the demographic model described above. The simulation is ended if the population either goes extinct $\left(N_{t}=0\right.$ for some $\left.t\right)$ 
(A)

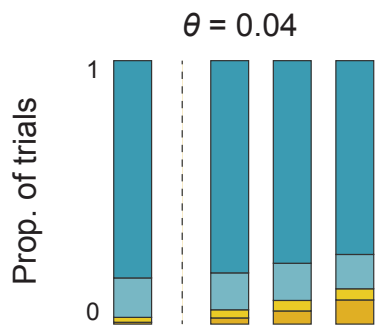

(B)

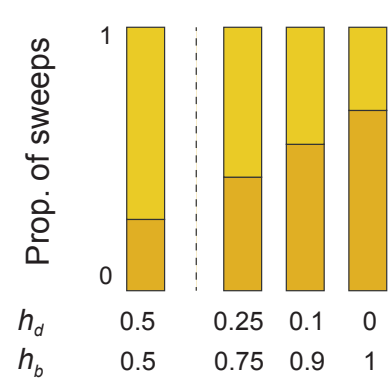

$\theta=0.4$
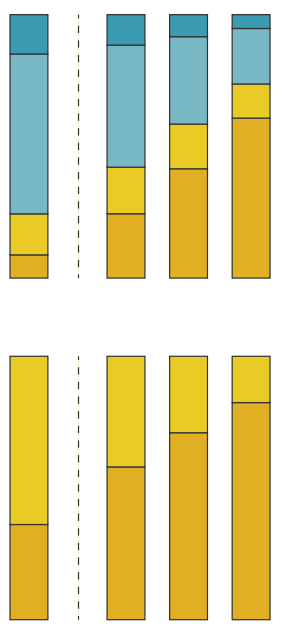

0.5

0.5

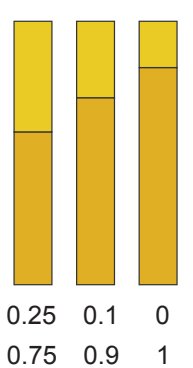

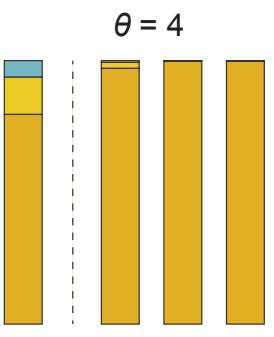

$s_{d}=0.01$

$s_{b}=0.01$

Figure 1: Expected dominance shifts increase the likelihood of a selective sweep and the proportion of selective sweeps that are soft. (A) The proportion of simulations that result in each of the four possible outcomes after the environmental change. The probability of a sweep (combined yellow and orange bars) increases with increasing severity of the focal allele's dominance shift. This effect is proportionately largest when the rate of mutational supply, $\theta=4 N u$, is small. (B) Among trials in which a sweep occurs, the proportion in which multiple copies from the standing variation account for the descendant copies at the time of completion of the sweep. The proportion of such 'soft sweeps' increases with the severity of the dominance shift, with this effect again proportionately largest for small values of $\theta$.

or re-attains its carrying capacity $\left(N_{t}=K\right.$ for some $\left.t\right)$. In the latter case, the population has been rescued. Although the population size is initially expected to decline because the $A$ allele is most common and $\left(1+r_{0}\right)\left(1-s_{2}\right)<1$, we allow for chance fluctuations against selection in these early generations by only classifying a simulation run as an example of evolutionary rescue if $N_{t}=K$ at least 10 generations after $T$. We do not allow for recurrent mutation after $T$.

In simulation runs where rescue is observed, we record: (i) The frequency of the $a$ allele. This is of particular interest because, with fitnesses specified as in the model above, classical theories of dominance predict that there should often be heterozygote advantage at the focal locus in the new environment (see Results), in which case evolutionary rescue will involve only a partial sweep of $a$. (ii) The number of ancestral copies of $a$ represented among descendant copies at the time of rescue. (iii) The haplotypic diversity among the population of $a$ alleles at the time of rescue, the metrics for which are described above. (iv) The number of generations taken for rescue; i.e., the smallest $t$ for which $N_{t}=K$ (with the requirement that $t>10$ ). (v) The minimum population size (i.e., how close the population came to extinction).

\section{Results}

\section{Expected dominance shifts increase the likelihood of a selective sweep.}

A primary effect of the expected shift in dominance of the focal allele from recessive deleterious to dominant beneficial is to increase the probability that a selective sweep will occur after the environmental change. This increase is especially pronounced when the rate of mutational supply, $\theta=4 N u$, is small (Fig. 1A). 
The reason for this increased probability of a sweep is straightforward. First, because the focal allele is recessive deleterious before the environmental shift, the mutation-selection-drift distribution is shifted towards there being more copies of the allele in the standing variation, relative to the case where the allele shows greater dominance in its deleterious effect (Fig. S1). Second, the fact that the focal allele is dominant beneficial after the environmental change means that Haldane's sieve does not hamper its initial chances of increasing in frequency and ultimately fixing. The first fact explains why the allele enjoys a higher probability of sweeping to fixation than a comparable allele that is dominant both before and after the environmental change; the second fact explains the same outcome with respect to an allele that is recessive both before and after the environmental change (Fig. S4).

\section{Expected dominance shifts increase the likelihood of soft vs. hard sweeps.}

A dominance shift of the focal allele also increases the probability, conditional on a selective sweep occurring, that the sweep will derive from multiple copies of the allele that were present in the standing variation (a soft sweep by our 'population definition') relative to just one (a hard sweep). This effect is clearly driven by the influence of dominance on the mutation-selection-drift distribution before the environmental change: when the allele is recessive deleterious before the environmental change, there are likely to be more copies present in the standing variation at the time of the environmental change, and so it is more likely that multiple copies will be involved in a subsequent sweep. The effect of dominance shifts in increasing the relative likelihood of soft vs. hard selective sweeps is especially noticeable for small values of $\theta$ (Fig. 1B).

Importantly, for a given value of $\theta$, the expected number of copies of the allele that were present as standing variation at the time of the environmental change differs conditional on a hard vs. a soft sweep subsequently occurring (Fig. S1) (Hermisson and Pennings 2017). That is, hard and soft sweeps tend to derive from different parts of the mutation-selection-drift distribution, underscoring the point that the full mutation-selection-drift distribution of $a$ before the environmental change - rather than just the mean of this distribution - must be considered to understand the population genetics of subsequent adaptation (Hermisson and Pennings 2017).

There has recently been much debate about the size of the parameter space under which soft sweeps prevail over hard sweeps (Messer and Petrov 2013; Jensen 2014; Hermisson and Pennings 2017; Harris et al. 2018). A corollary of the results above is that this parameter space is substantially expanded by the shifts in dominance predicted by the physiological and evolutionary theories of dominance.

Two parameters have been considered particularly relevant for the relative likelihood of hard vs. soft sweeps: the rate of mutational supply of the focal allele $(\theta)$, and the ratio of the focal allele's beneficial effect after the environmental change to its deleterious effect beforehand $\left(s_{b} / s_{d}\right)$. On the first, soft sweeps are relatively unlikely for small values of $\theta$ (Hermisson and Pennings 2017). However, with dominance shifts of the focal allele, soft sweeps can be relatively likely for values as low as $\theta \sim 0.01$ (Fig. 1, left; Fig. S2). For higher values of $\theta$, where soft sweeps predominate over hard sweeps even if the focal allele's dominance is constant across the environmental change, dominance shifts have a relatively small effect on the likelihood of soft versus hard sweeps (Fig. 1, right). On the second, higher values of $s_{b} / s_{d}$ clearly make soft sweeps from the standing variation more likely, but there is uncertainty about the precise values of $s_{b} / s_{d}$ for which soft sweeps are expected to predominate (Jensen 2014; Hermisson and Pennings 2017). We find that dominance shifts drastically reduce the $s_{b} / s_{d}$ values required for soft sweeps to be likely -indeed, soft sweeps can predominate over hard sweeps for values as low as $s_{b} / s_{d}=0.1$ in certain parameter regimes (Fig. S2), and can predominate more generally when $s_{b} / s_{d}=1$ (Fig. 1 ).

These effects are strongest when the shift in dominance of the focal allele is complete, but more modest shifts in dominance can also cause soft sweeps to have an appreciable likelihood relative to hard sweeps for low values of $\theta$ and $s_{b} / s_{d}$ (Figs. 1, S3). Note that, when soft sweeps are defined according to the stricter 'sample definition', they become less likely. However, dominance shifts nevertheless 
(A)

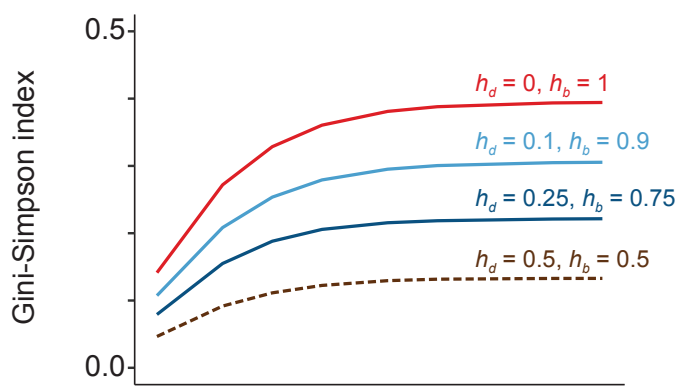

(B)

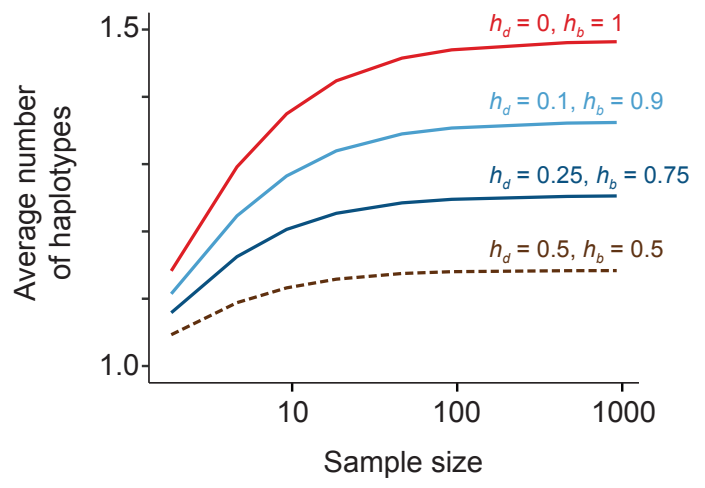

Figure 2: Expected dominance shifts increase (A) the allelic diversity and (B) the expected number of alleles involved in selective sweeps, causing a soft sweep to be detected more often in samples of alleles taken upon completion of the sweep. For the results displayed here, $s_{d}=0.01$ and $s_{b}=0.01$

increase the probability of soft sweeps according to this definition, and they are still possible for $\theta$ values as low as $\sim 0.01$ (Fig. S5).

\section{Expected dominance shifts lead to greater haplotypic diversity within soft sweeps.}

Expected dominance shifts also cause selective sweeps, when they do occur, to be mutationally (and therefore haplotypically) more diverse, according to the Gini-Simpson diversity index for various orders $n$ (Fig. 2A; see Methods). A practical consequence is that dominance shifts cause soft selective sweeps to be more detectable in small genomic samples, relative to the case where the focal allele's dominance remains constant across the environmental change. Similarly, under dominance shifts, a greater number of mutational lineages (and therefore haplotypes) are expected to be present in a sample of alleles taken at the time of completion of the sweep (Fig. 2B).

Thus, for the parameters considered in Fig. 2, and in a sample of 10 alleles taken upon completion of a sweep, a soft sweep would be detected only $11 \%$ of the time if the allele maintained a constant additive dominance before and after the environmental change (the expected number of haplotypes present in the sample is 1.11), but would be detected $33 \%$ of the time if the allele instead underwent the dominance shift predicted by the evolutionary and physiological theories of dominance (and the expected number of haplotypes in the sample would rise to 1.37).

\section{Expected dominance shifts decrease the importance of recurrent mutation versus standing variation for soft selective sweeps.}

Selective sweeps can derive from alleles that were present in the standing variation at the time of the environmental change, or from alleles that appeared by mutation after the environmental change (Pennings and Hermisson 2006; Hermisson and Pennings 2017). Recurrent mutation after the envi- 


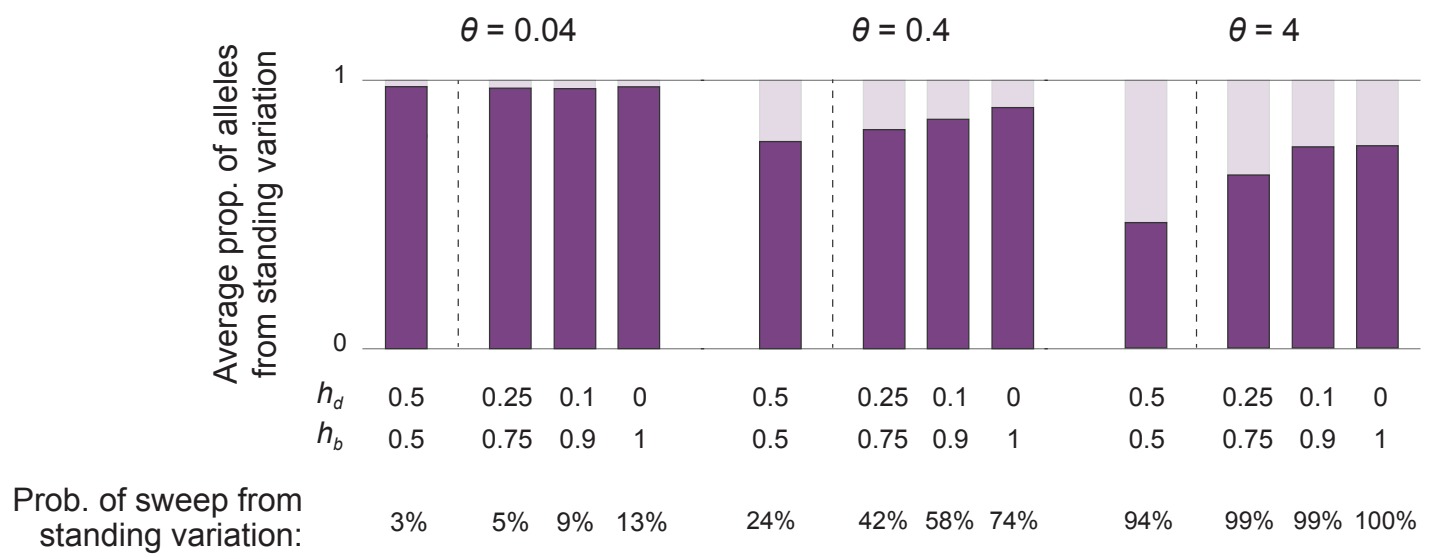

Figure 3: In soft sweeps sourced in part from the standing variation at the time of the environmental change, dominance shifts increase the representation of alleles from the standing variation relative to those produced by mutation after the environmental change. When $\theta$ is low, successful sweeps are dominated by alleles from the standing variation regardless of whether a dominance shift occurs (conditional on these sweeps involving at least one allele from the standing variation). In contrast, when $\theta$ is high, mutations arising after the environmental change have more opportunity to 'compete' with alleles from the standing variation before the sweep is completed, and dominance shifts strongly increase the representation of alleles from the standing variation in successful sweeps. For the results displayed here, $s_{d}=0.01$ and $s_{b}=0.01$.

ronmental change guarantees that a sweep will eventually occur, which narrows our interest to two questions.

First, how often is recurrent mutation necessary for a selective sweep to occur? This is equivalent to asking how often a selective sweep occurs in the absence of recurrent mutation, using alleles from the standing variation alone. We have addressed this question above, and have shown that dominance shifts substantially increase the probability that a selective sweep - and a soft sweep in particular - will occur from alleles that were present in the standing variation at the time of the environmental change. Second, in cases where a selective sweep occurs and alleles from the standing variation are involved, how large a role do mutations that occurred after the environmental change play in the sweep?

To answer this question, we incorporate recurrent mutation into our simulation setup (Methods). Previous theory has suggested that recurrent mutation should often play a leading role in selective sweeps compared to the standing variation (Pennings and Hermisson 2006; Hermisson and Pennings 2017). We find that a dominance shift of the focal allele increases the importance of the standing variation as a source of alleles in soft selective sweeps (Fig. 3). The reason is straightforward. Relative to the case where the allele is dominant (or semi-dominant) before and after the environmental change, a dominance shift of the focal allele does not alter the nature of selection acting on copies of it produced by recurrent mutation after the environmental change - in both scenarios, such copies arise at rate proportional to $\theta$ and are dominant beneficial. However, the dominance shift does increase the number of copies of the allele present in the standing variation at the time of the environmental change, improving the prospects of the standing variation as an allelic source of a subsequent sweep. Thus, the expected dominance shift increases the importance of the standing variation relative to recurrent mutation.

This effect of dominance shifts is most noticeable for high values of $\theta$ (e.g., $\theta \sim 1$ ), where the large supply of new mutations immediately after the environmental change increases the chance that these new alleles will be incorporated into a successful sweep. In contrast, for small values of $\theta$ (e.g., $\theta \sim 0.01$ ), the mutational supply of alleles immediately after the environmental change is small, so that sweeps - when they do occur - mostly involve alleles from the standing variation irrespective of their dominance before and after the environmental change. 


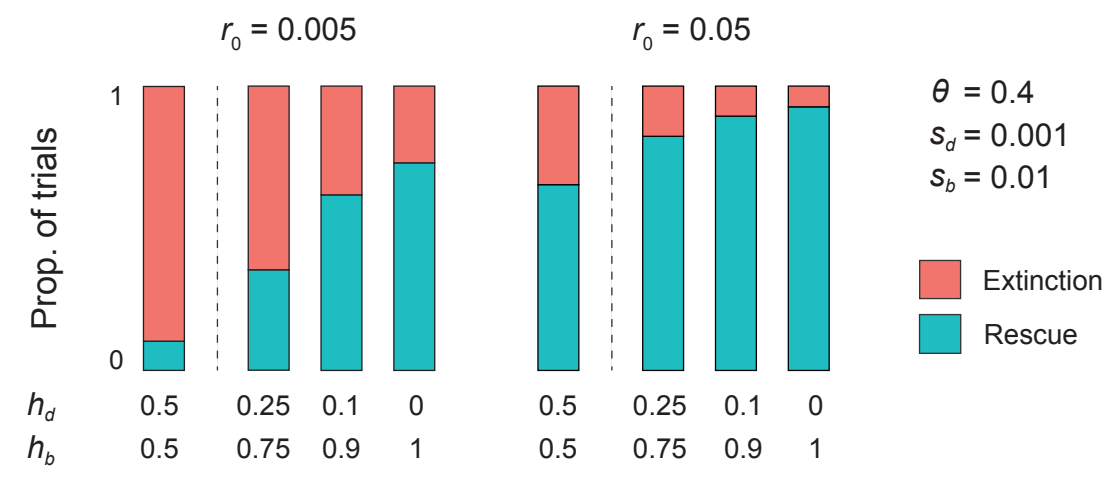

Average bottleneck (initial $N=10000$ ):

122

3498381401

Average time

until rescue (gens):

$1181 \quad 1048 \quad 874 \quad 727$
$879 \quad 2358 \quad 40275016$

$\begin{array}{llll}168 & 118 & 82 & 65\end{array}$

Figure 4: Expected dominance shifts increase the likelihood of evolutionary rescue. In cases where rescue does occur, it is more rapid, and the bottleneck suffered by the population less severe, when the rescuing allele undergoes a dominance shift. The likelihood of evolutionary rescue is especially increased by dominance shifts, relative to the case of constant dominance, when the population has a low intrinsic growth rate $r_{0}$ (left panel). However, the speed of rescue is especially increased by dominance shifts, and the population bottleneck made especially less severe, when $r_{0}$ is greater (right panel).

\section{Expected dominance shifts increase the likelihood of evolutionary rescue.}

We have thus far assumed a constant population size, both before and after the environmental change. However, in many cases of interest, the relative benefit enjoyed by the mutant allele $a$ after the environmental change will be due in part to a reduced absolute fitness of the wild-type allele $A$ in the new environment, such that a population fixed for $A$ would go extinct. In such cases, a selective sweep of $a$ might be required for the population to recover in size (i.e., to be 'rescued').

Incorporating into our model the dependence of population size on mean absolute fitness (Methods), we find that a dominance shift of $a$ increases the probability of evolutionary rescue, relative to the case where the dominance of $a$ remains constant across the environmental change (Fig. 4). Moreover, in cases where rescue does occur, the dominance shift of $a$ reduces the severity of the bottleneck suffered by the population, and allows the population to re-attain its prior size more rapidly (Fig. 4).

An interesting implication of expected dominance shifts in our model of evolutionary rescue is that heterozygote advantage after the environmental shift should be a common outcome. In this model, $a$ is associated with a relatively small absolute fitness cost in both the pre- and post-change environments, while $A$ is associated with a relatively large absolute fitness cost in the post-change environment. Therefore, $a$ is relatively disadvantageous before the environmental change, but relatively advantageous after (making possible evolutionary rescue). When $a$ is recessive with respect to the absolute fitness disadvantage it induces before the environmental change, but dominant with respect to the absolute fitness disadvantage induced by $A$ after the environmental change, then $A a$ heterozygotes suffer neither of the absolute fitness reductions induced by the two alleles in the post-change environment, and therefore enjoy the highest relative fitness. In such cases, when evolutionary rescue does occur, it is expected to proceed via a partial sweep of $a$, resulting in a balanced polymorphism at the focal locus.

\section{Case study: Adaptation at the Ace locus.}

To demonstrate the empirical relevance of expected dominance shifts for selective sweeps, we consider their importance for a well-studied case of adaptation to a new environment in insects: adaptation at 
the Ace locus in response to pesticide use.

Karasov et al. (2010) collected sequence data at the Ace locus from pesticide-resistant and pesticidesensitive strains of Drosophila melanogaster. Comparing the sequences of resistant alleles, they inferred multiple haplotypes, i.e., a soft selective sweep by our 'sample definition'. Since the point mutations that confer resistance are known in this case (Menozzi et al. 2004), Karasov et al. (2010) were able to calibrate the standard selective sweeps model using the point mutation rate of $D$. melanogaster, the species' traditionally quoted effective population size $\left(N_{e} \sim 10^{6}\right)$, and known fitness parameters for resistant alleles at the Ace locus, but assuming the dominance of resistant alleles to be constant before and after the onset of pesticide use. Karasov et al. (2010) found that, under these parameters: (i) a sweep involving the standing variation would have been unlikely; (ii) a sweep seeded by mutation after the onset of pesticide use would likely have been hard.

To reconcile the selective sweeps model with their observation of a soft sweep at the Ace locus, Karasov et al. (2010) proposed that the relevant effective population size for recent adaptation (such as at the Ace locus) would not be based on the long-term demography of the species (as the traditional effective population size is), but would instead depend on more recent demography, owing to the short timescale over which the relevant adaptation has occurred. Because D. melanogaster has undergone a recent population expansion (Thornton and Andolfatto 2006), an effective population size based on recent demography would be substantially larger than the long-term effective population size. Substituting into the selective sweeps model an effective population size two orders of magnitude larger than the traditionally quoted quantity, Karasov et al. (2010) found that a multi-haplotype soft sweep - as observed in their data - would be the expected outcome.

Our results suggest another factor that would help to reconcile the surprisingly high haplotypic diversity of resistant alleles at the Ace locus with the classic model of a selective sweep: a dominance shift of resistant alleles, from recessive deleterious in pesticide-free environments to dominant beneficial in environments of pesticide use. Indeed, empirical evidence suggests that resistant alleles at Ace in insects have exactly this pattern of fitness dominances in the two environments (Bourguet and Raymond 1998; Charlesworth 1998; Zhang et al. 2015). To understand what effect such dominance shifts might have on expected diversity among resistant alleles, we employ the same mutation and fitness parameters as Karasov et al. (2010), and estimate the likelihood of a sweep, the likelihood that the sweep is soft (by both the population and sample definitions), and the expected haplotypic diversity within a sweep, for various degrees of dominance shift [including no shift, as considered by Karasov et al. (2010)] and for various effective population sizes.

First, we study the case of $\theta=0.04$, a value that corresponds approximately to the traditional value of the effective population size in D. melanogaster $\left(N_{e} \approx 10^{6}\right)$. First, we find that the probability of adaptation from the standing variation is extremely small when there is no dominance shift of the resistant allele $\left(h_{d}=h_{b}=0.5\right)$ (Fig. 5A), consistent with Karasov et al. (2010). In addition, in the rare cases where a sweep does occur from the standing variation, the sweep usually involves only one copy of the resistant allele - and almost always only one haplotype (Fig. 5B,C). Under a full dominance shift of the resistant allele $\left(h_{d}=0 ; h_{b}=1\right)$, the probability of adaptation from the standing variation increases substantially, but remains small, when $\theta=0.04$. When a sweep does occur, it now more often uses multiple copies of the resistant allele from the standing variation (Fig. 5B), but still typically involves only one haplotype (Fig. 5C). Thus, in this case, sweeps would typically be soft by the population definition, but these soft sweeps would seldom be detectable in genomic data.

We now study the case of $\theta=0.4$, corresponding to a ten-fold higher effective population size than the traditional quantity for $D$. melanogaster, but a ten-fold lower value than that posited by Karasov et al. (2010) to explain the allelic diversity they observed at Ace. When the resistant allele maintains a constant additive dominance across the pre- and post-pesticide-use environments, adaptation from the standing variation remains unlikely in this case (Fig. 5A). Furthermore, when sweeps do occur, they typically make use of only one allele, and one haplotype, from the standing variation (Fig. 5B,C). 
(A)

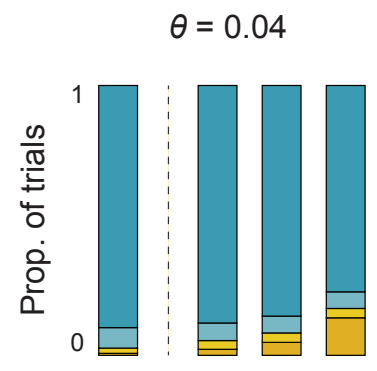

(B)

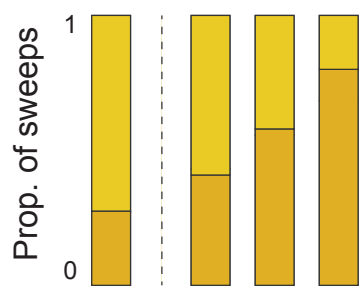

(C)

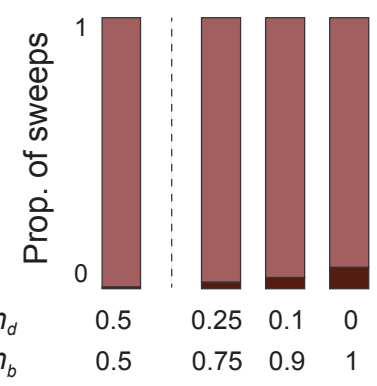

$\theta=0.4$

$s_{d}=0.1$

$s_{b}=0.1$

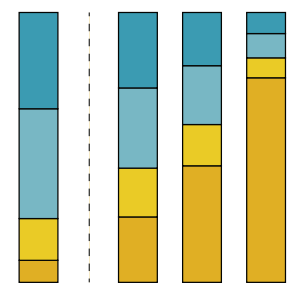

$\square$ No standing variation

Failed sweep

Hard sweep

Soft sweep
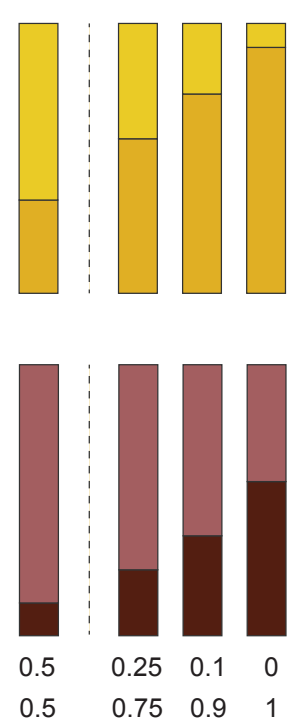

Single-haplotype sweep

? Multi-haplotype sweep

Figure 5: In response to the use of organophosphate pesticides, a dominance shift of resistant alleles at the Ace locus increases (A) the likelihood of a sweep from the standing variation, (B) the likelihood that a sweep from the standing variation is soft, and (C) the likelihood that multiple distinct haplotypes are involved in a sweep. These probabilities are displayed for values of $\theta$ corresponding to the long-term effective population size of Drosophila melanogaster (left panels) and, following the logic of Karasov et al. (2010), an increased estimate of the relevant effective population size based on more recent demography of the species (right panels). With this modestly revised estimate of the relevant effective population size, the soft sweep that Karasov et al. (2010) inferred from sequence data would be the expected outcome under a strong dominance shift of resistant alleles; moreover, alleles from the standing variation alone would usually be sufficient to furnish an empirically detectable soft sweep.

However, when the resistant allele undergoes a full dominance shift, adaptation from the standing variation becomes the expected outcome (Fig. 5A). Now, when a sweep does occur, it almost always makes use of multiple copies of the allele from the standing variation (Fig. 5B; a soft sweep by the population definition), and more often than not involves multiple haplotypes (Fig. 5B), rendering the soft sweep empirically detectable in genomic data.

The results described above ignore the possibility of recurrent appearance of resistant alleles by mutation after the onset of pesticide use. Therefore, they demonstrate that the diversity observed among swept alleles can be furnished entirely by the standing variation at the time of the environmental shift. To investigate the relative importance of the standing variation versus recurrent mutation, we allow for a positive rate of mutation to resistant alleles after the onset of pesticide use, equal to the mutation rate beforehand. Since, when adaptation from the standing variation does not occur, recurrent mutation will eventually furnish alleles that sweep to fixation, we focus on the relative importance of the standing variation versus recurrent mutation in cases where the standing variation does supply some of the alleles involved in a selective sweep. The 'unconditional' importance of the 
standing variation versus recurrent mutation can then be calculated by simply weighting according to the probability that a sweep does occur that involves alleles from the standing variation.

When $\theta=0.04$, sweeps that involve alleles from the standing variation seldom also involve an appreciable frequency of alleles produced by recurrent mutation, especially under a dominance shift of the resistant allele (Fig. S6). However, given the rarity of sweeps that do involve alleles from the standing variation in this case (Fig. 5A), adaptation in response to pesticide use must typically exclusively involve resistant alleles produced by mutation after the onset of pesticide use.

When $\theta=0.4$, it is still the case that sweeps involving alleles from the standing variation typically make use of few or no alleles produced by recurrent mutation (Fig. S6). This is especially so under a dominance shift of the resistant allele (Fig. S6). However, in contrast to the case where $\theta=0.04$, the increased frequency of sweeps that do involve alleles from the standing variation when $\theta=0.4$ (Fig. 5A) implies that the unconditional importance of recurrent mutation for adaptation in response to pesticide use is reduced. This holds whether there is a dominance shift or not. However, it is especially strong under a dominance shift because both (i) the importance of recurrent mutation in sweeps that involve the standing variation is smaller (Fig. S6), and (ii) sweeps that do involve the standing variation are more common (Fig. 5A).

In summary: (i) The genetic diversity observed by Karasov et al. (2010) at the Ace locus in D. melanogaster can be explained with a more modestly revised estimate of the relevant effective population size of this species when the dominance shift that resistant alleles have empirically been shown to exhibit is taken into account. (ii) For the associated value of $\theta$, and with a dominance shift of the resistant allele, the standing variation at the time of the environmental change is itself capable of furnishing the alleles involved in a subsequent empirical soft selective sweep. (iii) Moreover, in this case, when a soft selective sweep does occur, it is expected to predominantly involve alleles from the standing variation, rather than alleles produced by mutation after the environmental change.

\section{Discussion}

More than a century of research on the physiological and evolutionary bases of allelic dominance has been centered around, and has generated explanations for, the fact that beneficial alleles tend to be dominant while deleterious alleles tend to be recessive (Bourguet 1999; Falk 2001). Here, we have explored the implications of this pattern of fitness dominance for the genetics of adaptation to a new environment, with an allele that was deleterious in the old environment becoming beneficial in the new environment. This model is basic to the selective sweeps literature, but has typically been studied under the assumption that the fitness dominance of the focal allele is constant across the environmental change. This is contrary to the prediction of the physiological and evolutionary theories of dominance, which suggest that the allele should instead often shift from recessive deleterious before the environmental change to dominant beneficial after. We have shown that, relative to the case where the allele maintains a constant fitness dominance, the expected shift in dominance: (i) increases the probability of adaptation from the standing variation that was present at the time of the environmental shift; (ii) increases the probability that multiple alleles from the standing variation will contribute to adaptation, and that these alleles will lie on different haplotypes; (iii) increases the probability that a soft sweep from the standing variation will be detectable in small genomic samples; (iv) increases the importance of the standing variation relative to subsequent mutation for eventual adaptation to the new environment; (v) increases the probability of evolutionary rescue when the change of environment threatens the viability of the population. 


\section{Connections to previous theory}

While most of the prior literature on selective sweeps in a new environment has assumed constant dominance of the relevant alleles, a notable exception is Orr and Betancourt (2001), who consider the case of an allele that transitions from deleterious to beneficial across an environmental change, allowing for the possibility that the allele's fitness dominance shifts across the environmental change as well. They find that the probability that copies of the allele segregating in the standing variation at the time of the environmental change go on to fix is modulated by the ratio of the dominance of the focal allele in the new environment to its dominance in the old environment $\left[h_{b} / h_{d}\right.$; Eq. 19 in Orr and Betancourt (2001)]. However, they argue that, because 'it is hard to see why [dominance] shifts would be systematic in direction', the dominance values of alleles across environmental shifts will tend to cancel each other out on average, and thus have no systematic effect on the probability of adaptation from the standing variation.

Orr and Betancourt's calculations assume that, at the time of the environmental change, the allele's copy number equals the expectation of its mutation-selection distribution from before the environmental change, although they do compare their results against numerical computations based on the full mutation-selection-drift distribution. Hermisson and Pennings (2005) carry out an analytical calculation of the probability of adaptation from the standing variation, taking into account the full mutation-selection-drift distribution of the allele. They find that, unless selection against the allele before the environmental change is weak, the probability of adaptation from the standing variation is a function of $h_{b} / h_{d}$ [Eq. 8 in Hermisson and Pennings (2005)], echoing the result of Orr and Betancourt (2001). They conclude that, when the dominance of the allele does not shift across the environmental change, its value does not matter for the probability of adaptation from the standing variation.

We have argued, based on the physiological and evolutionary theories of dominance, that systematic dominance shifts of the alleles involved in adaptation to new environments are in fact expected - these alleles are predicted often to shift from recessive when deleterious to dominant when beneficial. These expected dominance shifts facilitate adaptation from the standing variation by increasing the presence of the allele in the population prior to the environmental change, and by rescuing the allele from Haldane's sieve after the environmental change. By incorporating the insights of the physiological and evolutionary theories of dominance into models of adaptation to new environments, we have shown that expected dominance shifts have a large impact not only on the probability of adaptation from the standing variation, but also on the genetic nature of this adaptation - in particular, whether it proceeds via hard or soft selective sweeps.

\section{When is a dominance shift expected?}

We have outlined two scenarios under which an environmental change can cause an allele to transition from deleterious to beneficial. In the first scenario, the phenotype that is primarily under selection does not change, but the direction of selection acting on the phenotype does. Since the same phenotype is under selection across the environmental change, the fitness dominance of an allele that affects the phenotype is not expected to shift appreciably. One context where this case is expected to be especially common is domestication, where a trait that was previously suppressed by breeders might suddenly become desired [e.g., various coat properties in domestic dogs (Cadieu et al. 2009)].

In the second scenario, the change in environment corresponds to a change in the phenotype that is primarily under selection. The focal allele is deleterious before the environmental change through its association with the old phenotype under selection, and beneficial after the environmental change through its association with the new phenotype under selection. The fitness dominance of the allele is then not constrained to remain constant across the environmental change, and, indeed, theories of dominance predict that the dominance of the allele should usually increase as it transitions from deleterious to beneficial. 
Environmental changes that generate selection on new phenotypes are, of course, expected to be common. We have discussed, as an example, the evolution of pesticide resistance at the Ace locus in Drosophila. In this case, the phenotype that was primarily under selection changed from 'intrinsic' enzymatic efficiency in the pesticide-free environment to the ability to inhibit pesticide binding once pesticides came into common use. As predicted by theories of dominance, resistant alleles at Ace switched from recessive deleterious to dominant beneficial upon the onset of pesticide use.

A similar situation can occur when a population is exposed to new diseases or parasites. A wellknown example of adaptation in humans involves the $\beta$-globin gene. Homozygotes for the 'sickle-cell' allele of this gene have characteristically misshapen red blood cells, and suffer from sickle-cell anemia, a severe blood disorder (Kwiatkowski 2005; Hedrick 2011). Heterozygotes produce functional red blood cells; the substantial deleterious effect of the allele is thus recessive (Kwiatkowski 2005). The sicklecell allele also offers protection against malarial infection for both heterozygotes and homozygotes (Kwiatkowski 2005; Hedrick 2011), leading to a situation of heterozygote advantage in environments where the disease is prevalent (because homozygotes still suffer the severe effects of sickle-cell anemia). Thus, as in the case of the Ace locus, the set of phenotypes under selection changes depending on the environment: in malaria-free environments, the phenotype under selection is 'intrinsic' function of red blood cells, while in environments where malaria is widespread, both intrinsic function and the ability to protect against malaria are under selection.

The sickle-cell variant of $\beta$-globin is one of a broader class of mutations in humans-including the variants causing $\alpha$ and $\beta$-thalassemia, glucose-6-phosphate dehydrogenase deficiency, and cystic fibrosis - that are associated with substantial fitness costs but also confer protection against some pathogen (Clegg and Weatherall 1999; Kwiatkowski 2005; Nielsen et al. 2007; Hedrick 2012). As predicted by theories of dominance, these variants display contrasting fitness dominances in pathogen-free and pathogen-affected environments. They are recessive deleterious in pathogen-free environments, where selection acts primarily on the diseases that they cause. In environments where the pathogens that they confer resistance against are prevalent, the fitness dominance of these variants characteristically shifts all the way to overdominance. This overdominance is predicted by our 'bottom-up' model of adaptation to a new environment, as employed in our analysis of evolutionary rescue, and is a consequence of the standard dominance patterns when there are two selectively independent phenotypes.

Environmental changes frequently expose populations to novel causes of selection (e.g., pesticides or pathogens in the examples set out above), and so often lead to shifts in the phenotypes that are primarily under selection. For this reason, shifts in the phenotypes under selection are also expected when populations colonize a new geographic area or expand into a new ecological niche. For alleles that affect both the old and new selected phenotypes, dominance shifts are expected. Thus, dominance shifts may play an important role in facilitating adaptation from the standing variation across a broad range of evolutionary and ecological contexts.

\section{Implications for the genetics of adaptation across the genome}

Our work has focused on the case of a single diploid locus, for which we have shown that the dominance shifts predicted by the evolutionary and physiological theories of dominance lead to an increased probability of adaptation to a new environment from the standing variation, and an increased probability that multiple alleles will be involved in this adaptation - i.e., an increased probability of a soft selective sweep. This result relies on the possibility of strong fitness dominance, and therefore points to differences across the genome in where hard vs. soft sweeps are likely to occur.

First, while autosomal loci are diploid in both sexes, and therefore subject to the patterns of the genetics of adaptation elucidated in this paper, X-linked loci in male-heterogametic systems and Zlinked loci in female-heterogametic systems are diploid in one sex but haploid in the other. At these sex-linked loci, alleles that become beneficial in a new environment cannot have been strongly recessive 
deleterious in the old environment, because they are hemizygously expressed in one sex. Therefore, we predict that soft sweeps from the standing variation should be less common at sex-linked loci than at autosomal loci. This prediction is, of course, complicated by other differences between autosomes and sex chromosomes, such as differences in their effective population size and the strength of selection on males vs. females (Vicoso and Charlesworth 2006).

Second, at a single diploid locus, the fitness effects of a deleterious allele can be 'masked' by the wild-type allele at the same locus, but when there are two (or more) copies of a locus, a deleterious allele can be masked by a wild-type allele at its locus or at the other locus. Therefore, we expect a deleterious allele at a duplicated locus to be more recessive than it would be in the single-locus case, since it has potentially more wild-type alleles to mask its deleterious effect. If the allele were later to become dominant beneficial, a soft sweep would then be an even more likely outcome than in the single-locus case, because the allele would undergo a more extreme dominance shift. This increased likelihood of a soft sweep would be further enhanced by the larger mutational target presented by the duplicated locus, i.e., a higher effective value of $\theta$.

Note that, if, after the change of selective environment, a sweep does occur at a duplicated locus, this would appear as neofunctionalization of the gene (Ohno 1970; Force et al. 1999). However, another source of apparent neofunctionalization is suggested by the the 'bottom up' model of dominance shifts that we used to study the particular case of evolutionary rescue. Under this model, heterozygote advantage is expected to be a common outcome of changes in the selective environment, leading to a partial selective sweep and subsequent stable polymorphism at the focal locus. In a sexual species, Mendelian segregation at the polymorphic locus causes the production of less fit homozygous genotypes, inducing a 'segregation load' that can select for duplication of the locus and fixation of the alternative alleles, one at each locus (Haldane 1954; Spofford 1969; Milesi et al. 2017). These two sources of neofunctionalization could be empirically distinguished if the timing of the duplication were either known a priori or inferable through the sequence divergence of the two gene copies. A case where the chronology of gene duplication is well established is the Ace locus in Culex pipiens (Labbé et al. 2007). In multiple populations subjected to pesticides, haplotypes that harbor a duplication of the Ace locus, with a copy each of the susceptible and resistant allele, are undergoing selective sweeps (Labbé et al. 2007; Alout et al. 2011; Milesi et al. 2017). The duplication is known to have occurred after the onset of pesticide use (Labbé et al. 2007), consistent with the second scenario for neofunctionalization outlined above.

\section{When are dominance shifts most important?}

We have shown that dominance shifts can lead to the frequent occurrence of soft selective sweeps in parameter regimes where they ordinarily would not be expected to occur. In particular, we have shown that dominance shifts can have a very strong impact on the genetics of adaptation when the focal allele is highly deleterious prior to the environmental change (Figs. 5, S2, S7). This is because, when selection against a deleterious allele is strong, its dominance has a large effect on the shape of its mutation-selection-drift distribution, which in turn determines the likelihood of a soft selective sweep from the standing variation once the allele becomes beneficial (Fig. S1). In contrast, if selection against the allele prior to the environmental shift is weak, there will usually be many copies present in the standing variation, regardless of its dominance, and so a soft selective sweep would be a common outcome in any case.

Our prediction that dominance shifts will be more influential when the focal allele is highly deleterious before the environmental change is complemented by a key prediction of the Wright-Kacser-Burns metabolic theory of dominance: the more deleterious a mutation is, the more recessive it usually will be (Kacser and Burns 1981; Phadnis and Fry 2005). This negative correlation between $h_{d}$ and $s_{d}$ has also been predicted by evolutionary theories of dominance [e.g., Manna et al. (2011)] and supported by empirical work in Drosophila (Simmons and Crow 1977; Charlesworth 1979) and yeast (Phadnis 
and Fry 2005; Agrawal and Whitlock 2011), and suggests that, precisely when dominance shifts are most influential - i.e., when the focal allele is highly deleterious before the environmental change - they should also be most pronounced (Fig. S7).

\section{Acknowledgments}

We are grateful to Graham Coop, Nate Edelman, Joachim Hermisson, Thomas Lenormand, Pleuni Pennings, Peter Wilton, and members of the Coop lab at UC Davis for helpful discussions, and to Graham Coop for comments that improved the manuscript. PM is supported by a Center for Population Biology postdoctoral fellowship. CV is supported by a Branco Weiss fellowship. This work was supported in part by the National Institute of General Medical Sciences of the National Institutes of Health (grants NIH R01 GM108779 and R35 GM136290 to G. Coop). The computations in this paper were run on the FASRC Odyssey cluster supported by the FAS Division of Science Research Computing Group at Harvard University.

\section{References}

Agrawal, A. F. and Whitlock, M. C. (2011). Inferences about the distribution of dominance drawn from yeast gene knockout data. Genetics, 187(2):553-566.

Alout, H., Labbé, P., Pasteur, N., and Weill, M. (2011). High incidence of ace-1 duplicated haplotypes in resistant Culex pipiens mosquitoes from Algeria. Insect Biochemistry and Molecular Biology, $41(1): 29-35$.

Barrett, R. D. H. and Schluter, D. (2008). Adaptation from standing genetic variation. Trends in Ecology \& Evolution, 23(1):38-44.

Bourguet, D. (1999). The evolution of dominance. Heredity, 83(1):1-4.

Bourguet, D., Lenormand, T., Guillemaud, T., Marcel, V., Fournier, D., and Raymond, M. (1997). Variation of dominance of newly arisen adaptive genes. Genetics, 147(3):1225-1234.

Bourguet, D. and Raymond, M. (1998). The molecular basis of dominance relationships: the case of some recent adaptive genes. Journal of Evolutionary Biology, 11(1):103-122.

Cadieu, E., Neff, M. W., Quignon, P., Walsh, K., Chase, K., Parker, H. G., VonHoldt, B. M., Rhue, A., Boyko, A., Byers, A., et al. (2009). Coat variation in the domestic dog is governed by variants in three genes. Science, 326(5949):150-153.

Charlesworth, B. (1979). Evidence against Fisher's theory of dominance. Nature, 278(5707):848-849.

Charlesworth, B. (1998). Adaptive evolution: the struggle for dominance. Current Biology, 8(14):R502-R504.

Clegg, J. B. and Weatherall, D. J. (1999). Thalassemia and malaria: new insights into an old problem. Proceedings of the Association of American Physicians, 111(4):278-282.

Donihue, C. M., Herrel, A., Fabre, A.-C., Kamath, A., Geneva, A. J., Schoener, T. W., Kolbe, J. J., and Losos, J. B. (2018). Hurricane-induced selection on the morphology of an island lizard. Nature, 560(7716):88-91.

Falk, R. (2001). The rise and fall of dominance. Biology and Philosophy, 16(3):285-323. 
Fisher, R. A. (1928). The possible modification of the response of the wild type to recurrent mutations. American Naturalist, 62(679):115-126.

Force, A., Lynch, M., Pickett, F. B., Amores, A., Yan, Y.-l., and Postlethwait, J. (1999). Preservation of duplicate genes by complementary, degenerative mutations. Genetics, 151(4):1531-1545.

Fournier, D. and Mutero, A. (1994). Modification of acetylcholinesterase as a mechanism of resistance to insecticides. Comparative Biochemistry and Physiology Part C: Pharmacology, Toxicology and Endocrinology, 108(1):19-31.

Fournier, D., Mutero, A., Pralavorio, M., and Bride, J.-M. (1993). Drosophila acetylcholinesterase: mechanisms of resistance to organophosphates. Chemico-Biological Interactions, 87(1-3):233-238.

Garud, N. R., Messer, P. W., Buzbas, E. O., and Petrov, D. A. (2015). Recent selective sweeps in North American Drosophila melanogaster show signatures of soft sweeps. PLoS Genetics, 11(2):e1005004.

Haldane, J. B. S. (1954). The Biochemistry of Genetics. George Allen \& Unwin, London.

Harris, R. B., Sackman, A., and Jensen, J. D. (2018). On the unfounded enthusiasm for soft selective sweeps II: Examining recent evidence from humans, flies, and viruses. PLoS Genetics, 14(12):e1007859.

Hedrick, P. W. (2011). Population genetics of malaria resistance in humans. Heredity, 107(4):283-304.

Hedrick, P. W. (2012). What is the evidence for heterozygote advantage selection? Trends in Ecology \& Evolution, 27(12):698-704.

Hermisson, J. and Pennings, P. S. (2005). Soft sweeps: molecular population genetics of adaptation from standing genetic variation. Genetics, 169(4):2335-2352.

Hermisson, J. and Pennings, P. S. (2017). Soft sweeps and beyond: understanding the patterns and probabilities of selection footprints under rapid adaptation. Methods in Ecology and Evolution, 8(6):700-716.

Hoffmann, F., Fournier, D., and Spierer, P. (1992). Minigene rescues acetylcholinesterase lethal mutations in Drosophila melanogaster. Journal of Molecular Biology, 223(1):17-22.

Hurst, L. D. and Randerson, J. P. (2000). Dosage, deletions and dominance: simple models of the evolution of gene expression. Journal of Theoretical Biology, 205(4):641-647.

Jensen, J. D. (2014). On the unfounded enthusiasm for soft selective sweeps. Nature Communications, $5(1): 1-10$.

Jost, L. (2006). Entropy and diversity. Oikos, 113(2):363-375.

Kacser, H. and Burns, J. A. (1981). The molecular basis of dominance. Genetics, 97(3-4):639-666.

Karasov, T., Messer, P. W., and Petrov, D. A. (2010). Evidence that adaptation in Drosophila is not limited by mutation at single sites. PLoS Genetics, 6(6):e1000924.

Keightley, P. D. (1996). A metabolic basis for dominance and recessivity. Genetics, 143(2):621.

Kwiatkowski, D. P. (2005). How malaria has affected the human genome and what human genetics can teach us about malaria. The American Journal of Human Genetics, 77(2):171-192. 
Labbé, P., Berthomieu, A., Berticat, C., Alout, H., Raymond, M., Lenormand, T., and Weill, M. (2007). Independent duplications of the acetylcholinesterase gene conferring insecticide resistance in the mosquito Culex pipiens. Molecular Biology and Evolution, 24(4):1056-1067.

Lenormand, T., Bourguet, D., Guillemaud, T., and Raymond, M. (1999). Tracking the evolution of insecticide resistance in the mosquito Culex pipiens. Nature, 400(6747):861-864.

Manna, F., Martin, G., and Lenormand, T. (2011). Fitness landscapes: an alternative theory for the dominance of mutation. Genetics, 189(3):923-937.

Menozzi, P., Shi, M. A., Lougarre, A., Tang, Z. H., and Fournier, D. (2004). Mutations of acetylcholinesterase which confer insecticide resistance in Drosophila melanogaster populations. BMC Evolutionary Biology, 4(1):4.

Messer, P. W. and Petrov, D. A. (2013). Population genomics of rapid adaptation by soft selective sweeps. Trends in Ecology \& Evolution, 28(11):659-669.

Milesi, P., Weill, M., Lenormand, T., and Labbé, P. (2017). Heterogeneous gene duplications can be adaptive because they permanently associate overdominant alleles. Evolution Letters, 1(3):169-180.

Mutero, A., Pralavorio, M., Bride, J.-M., and Fournier, D. (1994). Resistance-associated point mutations in insecticide-insensitive acetylcholinesterase. Proceedings of the National Academy of Sciences, 91(13):5922-5926.

Nielsen, R., Hellmann, I., Hubisz, M., Bustamante, C., and Clark, A. G. (2007). Recent and ongoing selection in the human genome. Nature Reviews Genetics, 8(11):857-868.

Ohno, S. (1970). Evolution by gene duplication. Springer-Verlag, Berlin.

Orr, H. A. and Betancourt, A. J. (2001). Haldane's sieve and adaptation from the standing genetic variation. Genetics, 157(2):875-884.

Pennings, P. S. and Hermisson, J. (2006). Soft sweeps II - molecular population genetics of adaptation from recurrent mutation or migration. Molecular Biology and Evolution, 23(5):1076-1084.

Phadnis, N. and Fry, J. D. (2005). Widespread correlations between dominance and homozygous effects of mutations: implications for theories of dominance. Genetics, 171(1):385-392.

Pritchard, J. K., Pickrell, J. K., and Coop, G. (2010). The genetics of human adaptation: hard sweeps, soft sweeps, and polygenic adaptation. Current Biology, 20(4):R208-R215.

Schrider, D. R. and Kern, A. D. (2017). Soft sweeps are the dominant mode of adaptation in the human genome. Molecular Biology and Evolution, 34(8):1863-1877.

Shi, M. A., Lougarre, A., Alies, C., Frémaux, I., Tang, Z. H., Stojan, J., and Fournier, D. (2004). Acetylcholinesterase alterations reveal the fitness cost of mutations conferring insecticide resistance. BMC Evolutionary Biology, 4(1):5.

Simmons, M. J. and Crow, J. F. (1977). Mutations affecting fitness in drosophila populations. Annual Review of Genetics, 11(1):49-78.

Spofford, J. B. (1969). Heterosis and the evolution of duplications. American Naturalist, 103(932):407432.

Stephan, W. (2019). Selective sweeps. Genetics, 211(1):5-13. 
Thornton, K. and Andolfatto, P. (2006). Approximate Bayesian inference reveals evidence for a recent, severe bottleneck in a Netherlands population of Drosophila melanogaster. Genetics, 172(3):16071619 .

Vicoso, B. and Charlesworth, B. (2006). Evolution on the X chromosome: unusual patterns and processes. Nature Reviews Genetics, 7(8):645-653.

Wright, S. (1934). Physiological and evolutionary theories of dominance. American Naturalist, 68(714):24-53.

Zhang, L. J., Jing, Y. P., Li, X. H., Li, C. W., Bourguet, D., and Wu, G. (2015). Temperaturesensitive fitness cost of insecticide resistance in Chinese populations of the diamondback moth Plutella xylostella. Molecular Ecology, 24(7):1611-1627. 


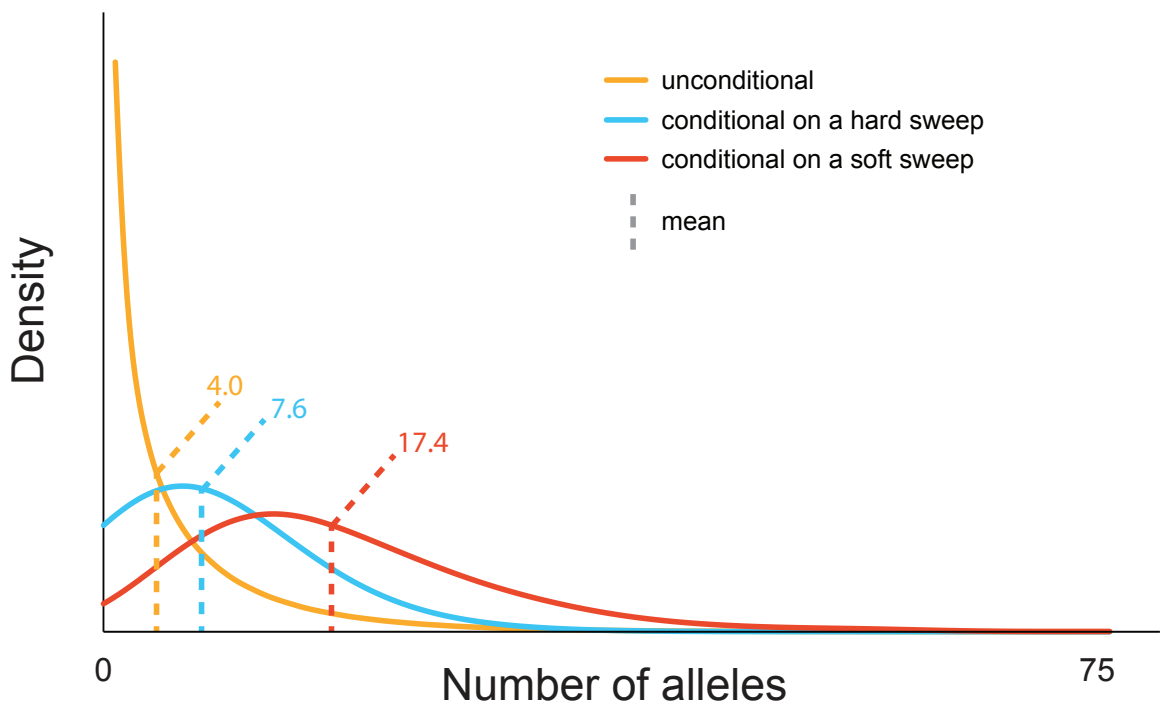

Figure S1: Hard and soft selective sweeps derive from different regions of the mutation-selection-drift distribution. The mutation-selection-drift distribution of a deleterious allele is shown in gold $\left[\theta=0.4\left(N=10^{4}\right.\right.$, $\left.\left.u=10^{-5}\right), s_{d}=0.1, h_{d}=0.5\right]$, while the distribution of starting allele counts that result in hard (blue) and soft (red) selective sweeps are overlaid $\left(s_{b}=0.1, h_{b}=0.5\right)$.

(A)

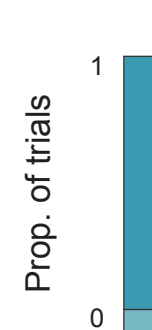

$\theta=0.04$

(B)

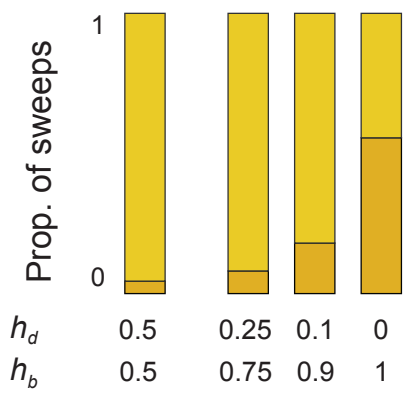

$\theta=0.4$

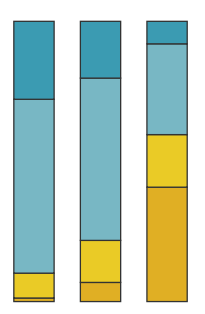

$s_{d}=0.1$

$s_{b}=0.01$

No standing variation

Failed sweep

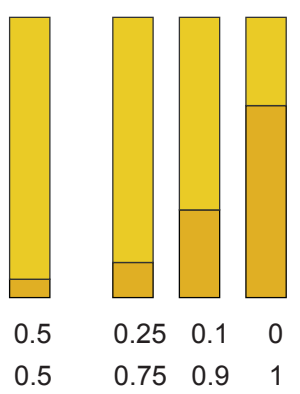

Hard sweep

Soft sweep

Figure S2: Expected dominance shifts can cause soft sweeps to predominate over hard sweeps even when $s_{b} / s_{d}$ is small. Previous work predicts that soft sweeps should be rare when $s_{b} / s_{d}<1$, owing to low levels of standing variation at the time of the environmental change and a high probability of stochastic loss of those copies that are present. However, if the focal allele undergoes a strong dominance shift $\left(h_{d} \approx 0, h_{b} \approx 1\right)$, soft sweeps can be more likely than hard sweeps despite small values of $s_{b} / s_{d}$. 
(A)

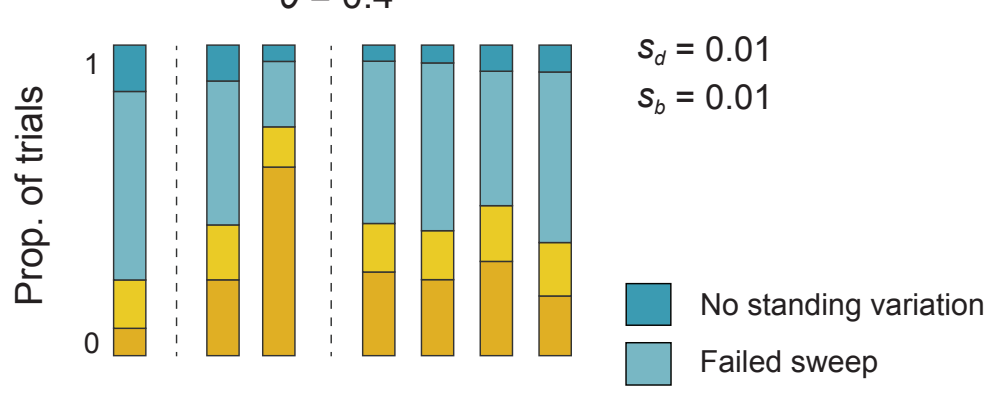

(B)

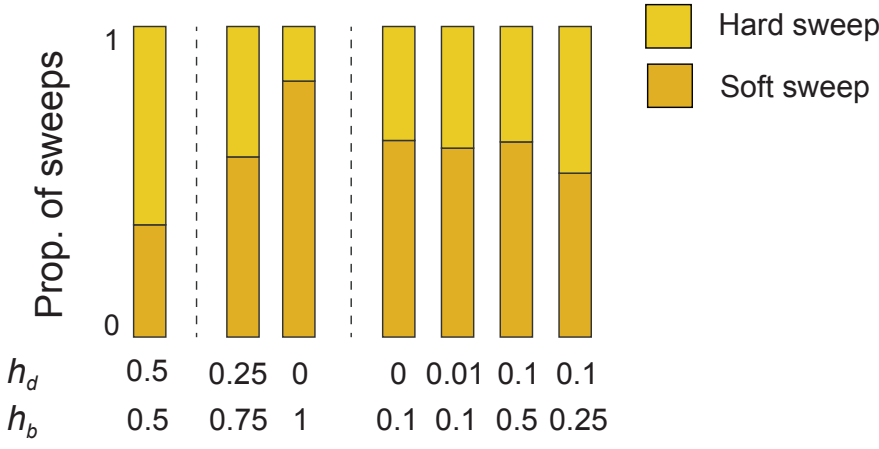

Figure S3: Expected dominance shifts strongly increase the likelihood of soft selective sweeps, even when the dominance of the focal allele after the environmental change is sub-additive $\left(h_{d}<h_{b}<0.5\right)$. However, such partial dominance shifts lead to smaller increases in the likelihood of a soft sweep, relative to complete dominance shifts $\left(h_{d} \approx 0, h_{b} \approx 1\right)$.

(A)

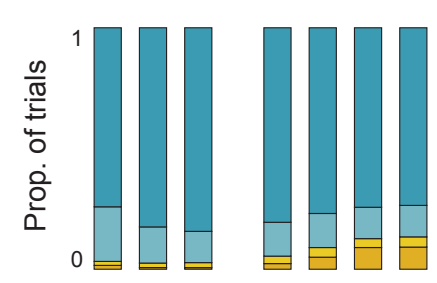

(B)

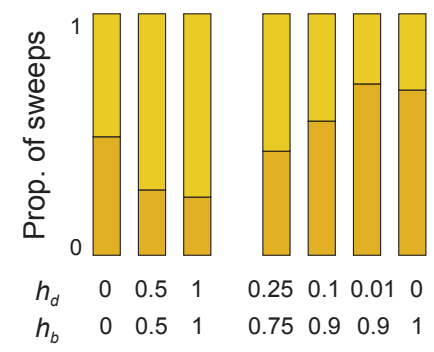

$\theta=0.4$
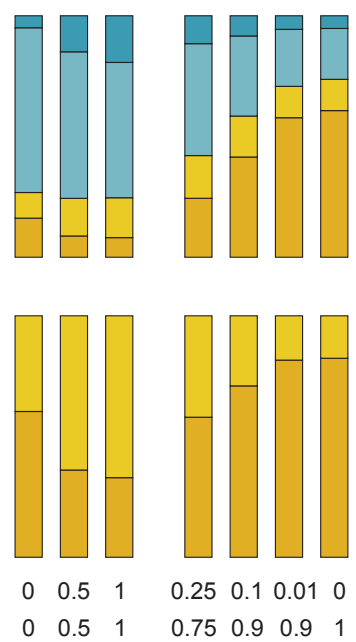

$\theta=4$

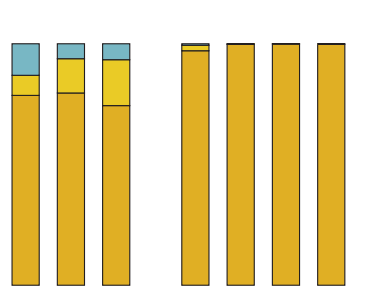

$s_{d}=0.01$

$s_{b}=0.01$

$\square$ No standing variation

Failed sweep

Hard sweep

Soft sweep

Figure S4: (A) Expected dominance shifts increase the likelihood of a selective sweep relative to scenarios in which the adaptive allele is constantly recessive, additive, or dominant. (B) Conditional on successful adaptation from the standing variation, expected dominance shifts increase the probability that multiple alleles are involved. When the allele maintains a constant dominance across the environmental shift $\left(h_{b}=h_{d}\right)$, the relative likelihood of soft sweeps is highest when it is fully recessive $\left(h_{d}=h_{b}=0\right)$. Compared to this case, a moderate dominance shift (e.g., $h_{d}=0.25, h_{b}=0.75$ ) leads to approximately the same relative likelihood of a soft selective sweep (and stronger dominance shifts increase this relative likelihood further). Moreover, since the probability of adaptation from the standing variation is lower for a constantly recessive allele than an allele undergoing a dominance shift (A), there will be a greater absolute number of soft sweeps in the latter case. 
(A)

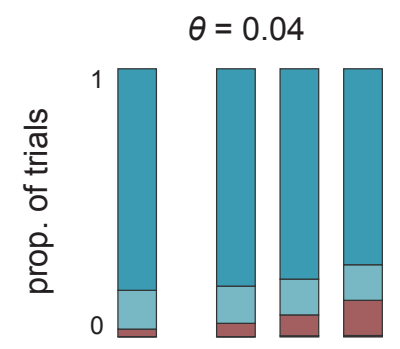

(B)

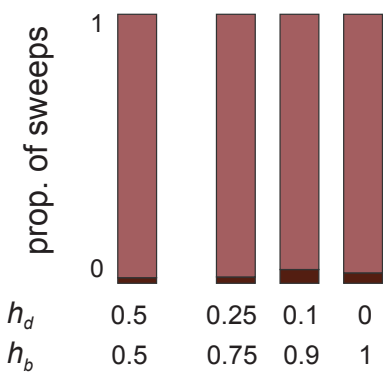

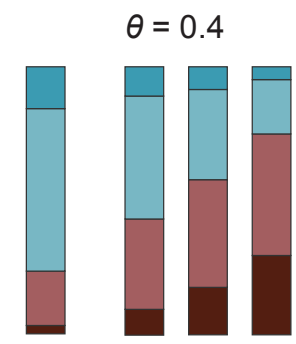

$s_{d}=0.01$

$s_{b}=0.01$

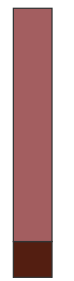

0.5

0.5

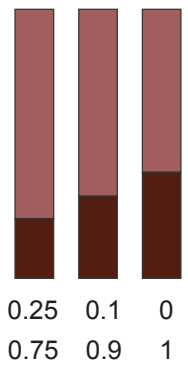

No standing variation

Failed sweep

Single-haplotype sweep

Multi-haplotype sweep

Figure S5: Expected dominance shifts increase the probability of soft sweeps with multiple independent mutational origins, and therefore multiple distinguishable haplotypes (soft sweeps by the 'sample definition').

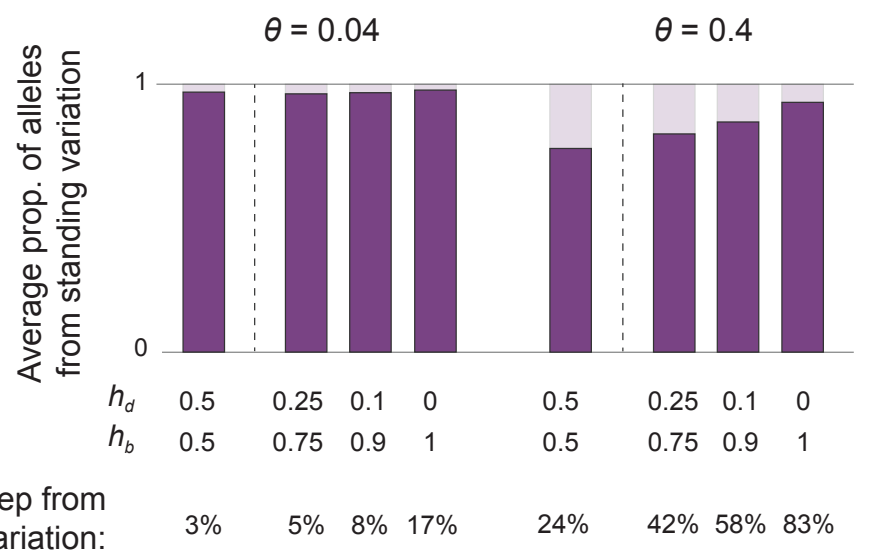

Figure S6: A dominance shift of resistant alleles at the Ace locus in Drosophila melanogaster after the onset of pesticide use increases the average representation of the standing variation, relative to recurrent mutation, among successful sweeps that involve some alleles from the standing variation. $\theta$ values correspond to the long-term effective population size of $D$. melanogaster (left) and an increased value based on more recent demography of the species (right), following the logic of Karasov et al. (2010). For the smaller value of $\theta$, if a sweep involves alleles from the standing variation, it is expected to be dominated by alleles from the standing variation regardless of whether a dominance shift occurs or not. In contrast, for the larger value of $\theta$, dominance shifts substantially increase the representation of standing variation among sweeps that involve some alleles from the standing variation. 

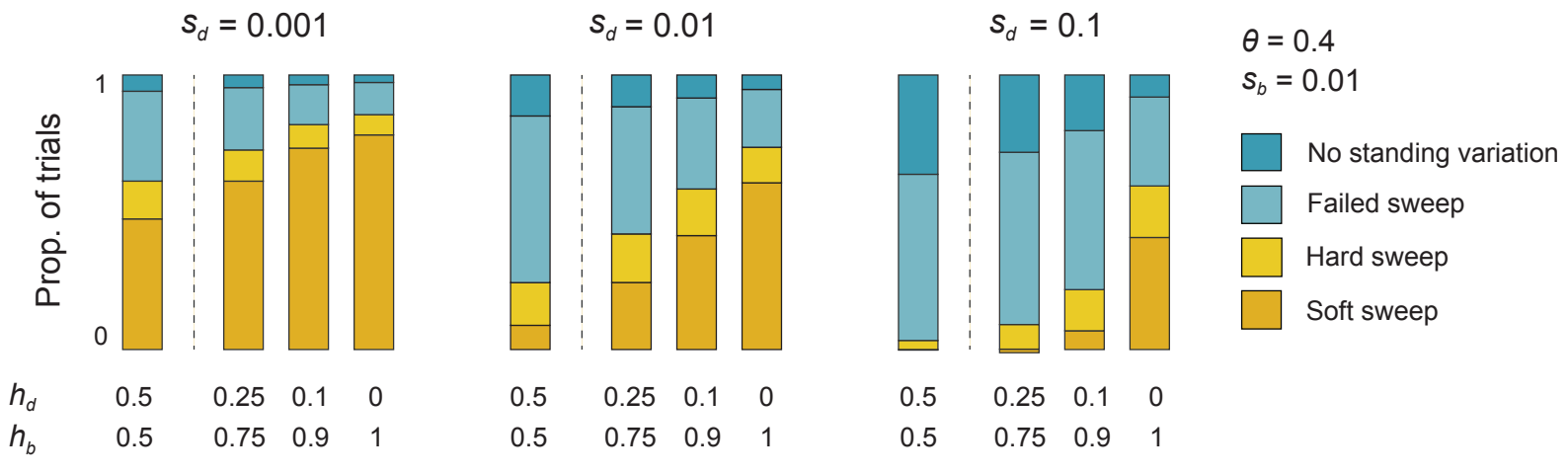

Figure S7: Expected dominance shifts have the largest proportional effect on the probability of adaptation from the standing variation, and the relative proportion of soft vs. hard sweeps, when selection against the focal allele prior to the environmental change is strong. Complementing this effect, theories of dominance predict a negative correlation between deleterious effect size and dominance, such that more deleterious alleles are typically more recessive. Thus, dominance shifts are expected to play a particularly important role in parameter regimes in which the focal allele is strongly selected against before the environmental change. 